\title{
Surgical Infection Prophylaxis for Left Ventricular Assist Device Implantation
}

\author{
Paul C. Walker, Pharm.D.,* Daryl D. DePestel, Pharm.D., ${ }^{\dagger}$ Nicholas A. Miles, Pharm.D.,* \\ and Preeti N. Malani, M.D., M.S.J.†, $\S$ \\ * Department of Pharmacy Services, University of Michigan Health System and College of \\ Pharmacy, Ann Arbor, Michigan; †Department of Medical Affairs, Cubist Pharmaceuticals, \\ Lexington, Massachusetts (current affiliation); $\ddagger$ Department of Internal Medicine, Division of \\ Infectious Diseases and Geriatric Medicine, University of Michigan Health System; and \\ $\S$ Veterans Affairs Ann Arbor Healthcare System and Geriatric Research Education and Clinical \\ Center (GRECC), Ann Arbor, Michigan
}

\begin{abstract}
AвSTRACt Background: Antimicrobial prophylaxis is commonly administered to patients undergoing left ventricular assist device (LVAD) surgeries to prevent infectious complications. However, optimal surgical infection prophylaxis (SIP) for LVAD surgeries is not well defined. Methods: We conducted an electronic survey to characterize LVAD SIP used at different centers performing LVAD implantation. Results: Responses were received from 23 of 85 centers (27\%). Of 21 centers that provided usable data about their LVAD SIP regimens for nonpenicillin allergic patients, $42.9 \%$ reported using a four-drug regimen (three antibiotics plus fluconazole), 23.8\% reported using a three-drug regimen (three antibiotics or two antibiotics plus fluconazole), $23.8 \%$ reported using a regimen of two antibiotics, and $9.5 \%$ reported using vancomycin alone. A similar pattern was observed among SIP regimens for penicillin-allergic patients. Criteria for discontinuation of SIP and use of decolonization strategies also varied widely across centers. Conclusions: Our results demonstrate wide variability in LVAD SIP regimens and underscore the lack of consensus regarding best practice. doi: 10.1111/j.1540-8191.2011.01262.x (J Card Surg 2011;26:440-443)
\end{abstract}

\section{INTRODUCTION}

Left ventricular assist device (LVAD) use continues to burgeon both as a bridge to heart transplantation as well as longer term "destination therapy." ${ }^{1}$ Infection remains the most serious complication of LVAD support and a leading cause of mortality, morbidity, and decreased quality of life. ${ }^{2}$ Data from the 2006 to 2009 interagency registry for mechanical circulatory support (INTERMACS) identified infection as the cause of $16 \%$ of deaths post-LVAD implantation, second only to heart failure. Previous reports have described infection rates between $13 \%$ and $80 \%$ among LVAD recipients. ${ }^{3}$

Prevention of LVAD infection using appropriate surgical infection prophylaxis (SIP) is especially critical among patients undergoing implantation for destination therapy since successful treatment of a serious

Conflict of interest: All authors report no conflicts of interest related to this work. Financial support: none.

Address for corespondence: Paul C. Walker, Pharm.D., Department of Pharmacy Services B2D301, University of Michigan Health System, Ann Arbor, MI 48109-0008. Fax: (734) 936-7027; e-mail: pcwalker@umich.edu device infection generally requires prompt removal followed by transplantation. ${ }^{3}$ Despite well-founded concerns for device-associated infection, an optimal SIP regimen is not well defined. Although current guidelines recommend limiting the duration of prophylaxis to no longer than 48 hours after cardiac surgery, these general guidelines are difficult to apply to LVAD implantation. ${ }^{4}$ Patients undergoing LVAD placement are clinically distinct from individuals undergoing scheduled cardiac procedures such as valve replacement or coronary artery bypass grafting. In particular, patients who require LVAD support are usually critically ill often with multiple indwelling lines and catheters. Many spend an extended period of time in an intensive care unit (ICU) prior to surgery and a large portion require delayed sternal closure and transfusion of blood products. These and other factors increase the risk of infection well above the rates associated with general cardiac surgeries. ${ }^{3}$

Despite considerable risk, there is a lack of consensus on the best approach to SIP for LVADs; practices can vary greatly from center to center. The prolonged use of antimicrobial agents is not therapeutically neutral. Besides toxicities and costs, patients receiving 
antimicrobials are at increased risk of Clostridium difficile infection as well as subsequent infection with antimicrobial-resistant organisms. ${ }^{5}$ Such acquisition of antimicrobial resistance not only impacts the patients receiving treatment, but also represents a broader challenge for infection control and antimicrobial stewardship programs. At our institution, the duration and choice of agents used for SIP has evolved since the first LVAD was implanted in 1996. In particular, the duration of vancomycin prophylaxis has decreased significantly. 6

With increasing numbers of devices being placed, LVAD SIP practices deserve closer attention. Given these concerns, we conducted a brief survey to evaluate SIP practices at different centers performing LVAD implantation.

\section{SURGICAL INFECTION PROPHYLAXIS IN LVADS}

\section{METHODS}

Centers for Medicaid and Medicare Services approved heart transplant centers were contacted to identify a clinical pharmacist who could complete a questionnaire about LVAD SIP regimens (http://www.cms.gov/CertificationandComplianc/20_

Transplant.asp). In October 2009, the questionnaire was sent electronically to these pharmacists. The survey recorded basic information about the center's LVAD program (annual volume, date of inception, etc.) along with characteristics of the preferred SIP regimens. Descriptive statistics were used to characterize responses. This study was approved by University of Michigan Medical School's Institutional Review Board.

\section{TABLE 1}

Reported Surgical Infection Prophylaxis Regimens for Left Ventricular Assist Device Placement

\begin{tabular}{|c|c|c|c|c|}
\hline & & & & Number of Respondents \\
\hline \multicolumn{5}{|c|}{ SIP Regimens for Nonpenicillin Allergic Patients* } \\
\hline \multicolumn{5}{|c|}{ One-drug Regimen } \\
\hline Cefazolin & & & & 1 \\
\hline Vancomycin & & & & 1 \\
\hline \multicolumn{5}{|c|}{ Two-drug Regimen } \\
\hline Vancomycin & Cefazolin & & & 2 \\
\hline Vancomycin & Cefuroxime & & & 1 \\
\hline Vancomycin & Ceftazidime & & & 1 \\
\hline Vancomycin & Quinolone & & & 1 \\
\hline \multicolumn{5}{|c|}{ Three-drug Regimen } \\
\hline Vancomycin & Pip/Tazo & Fluconazole & & 1 \\
\hline Vancomycin & Rifampin & Quinolone & & 1 \\
\hline Vancomycin & Fluconazole & Ciprofloxacin & & 1 \\
\hline Vancomycin & Fluconazole & Cefepime & & 1 \\
\hline TMP/SMX & Rifampin & Fluconazole & & 1 \\
\hline \multicolumn{5}{|c|}{ Four-drug Regimen } \\
\hline Vancomycin & Rifampin & Fluconazole & Cefazolin & 2 \\
\hline Vancomycin & Quinolone & Fluconazole & Cefepime & 1 \\
\hline Vancomycin & Rifampin & Fluconazole & Cefepime & 1 \\
\hline Vancomycin & Rifampin & Fluconazole & Quinolone & 1 \\
\hline Vancomycin & Rifampin & Quinolone & Fluconazole & 3 \\
\hline Vancomycin & Rifampin & Рip/Tazo & Fluconazole & 1 \\
\hline \multicolumn{5}{|c|}{ Sip Regimens for Penicillin-allergic Patients ${ }^{\dagger}$} \\
\hline \multicolumn{5}{|c|}{ One-drug Regimen } \\
\hline Vancomycin & & & & 3 \\
\hline \multicolumn{5}{|c|}{ Two-drug Regimen } \\
\hline Vancomycin & Aztreonam & & & 1 \\
\hline Vancomycin & Quinolone & & & 2 \\
\hline \multicolumn{5}{|c|}{ Three-drug Regimen } \\
\hline TMP/SMX & Rifampin & Fluconazole & & 1 \\
\hline Vancomycin & Quinolone & Fluconazole & & 2 \\
\hline Vancomycin & Levofloxacin & Aztreonam & & 1 \\
\hline Vancomycin & Fluconazole & Aztreonam & & 1 \\
\hline Vancomycin & Rifampin & Levofloxacin & & 1 \\
\hline \multicolumn{5}{|c|}{ Four-drug Regimen } \\
\hline Vancomycin & Quinolone & Fluconazole & Cefepime $\ddagger$ & 1 \\
\hline Vancomycin & Rifampin & Fluconazole & ID consult for cefepime substitution & 1 \\
\hline Vancomycin & Rifampin & Quinolone & Fluconazole & 6 \\
\hline
\end{tabular}

*Number of respondents providing data $=21$.

$\dagger$ Number of respondents providing data $=20$.

‡Unable to clarify with respondent.

$\mathrm{Pip} / \mathrm{Tazo}=$ piperacillin/tazobactam, TMP/SMX = trimethoprim/sulfamethoxazole. 


\section{RESULTS}

We received responses to the electronic survey from 23 of 85 transplant centers (27\%). Most responding centers were either university hospitals (56.5\%) or hospitals closely affiliated with an academic institution (30.4\%). Nine institutions (39.1\%) had been implanting LVADs for $>10$ years; six $(26.1 \%)$ had $<5$ years of experience. In terms of operative volume in 2008 , six $(26.1 \%$ ) centers implanted > 20 LVADs in 2008; another six reported 10 to 20 devices for 2008. All but two centers reported a formal SIP policy for LVAD placement (although both centers reported that they were actively developing a policy).

SIP regimens varied widely across institutions (Table 1). Twenty-one institutions provided usable data about their LVAD SIP regimens for nonpenicillinallergic patients. Nine institutions (42.9\%) reported using a four-drug regimen consisting of three antibiotics plus fluconazole. Most commonly, these regimens included vancomycin, a cephalosporin or quinolone, and rifampin. Five institutions $(23.8 \%)$ reported using a three-drug regimen consisting of three antibiotics or two antibiotics plus fluconazole. Another five institutions $(23.8 \%)$ reported a two-drug antibiotic regimen and two institutions (9.5\%) used vancomycin alone. Similar patterns were observed among the 20 institutions providing usable data for penicillin-allergic patients; four-drug, three-drug, two-drug, and single-drug antimicrobial regimens were used among 40\%, 30\%, $15 \%$, and $15 \%$ of institutions, respectively.

Thirteen centers $(61.9 \%)$ reported using some type of decolonization strategy prior to surgery. Specific approaches included mupirocin to the nares (12 of 13) and/or chlorhexidine washes (6 of 13), with five centers using both mupirocin and chlorhexidine.

Criteria for discontinuation of SIP also varied. Of the 20 institutions providing data, nine (45\%) reported discontinuing antimicrobials at 48 hours postoperatively; one center discontinued therapy at 24 hours postoperatively, another at 72 hours. Other criteria included: after six doses; at the attending physician's discretion; after sternal closure; when chest tubes are removed; automatically stopped, but criteria not specified.

\section{DISCUSSION}

The above results suggest wide variability in LVAD SIP regimens among centers responding to our electronic survey. Several centers reported using the SIP protocol outlined in the randomized evaluation of mechanical assistance therapy for congestive heart failure (REMATCH) trial including vancomycin, levofloxacin, rifampin, and fluconazole along with mupirocin. ${ }^{1}$ Criteria for stopping SIP varied even more with less than half of institutions routinely discontinuing SIP at 48 hours postoperatively.

Despite the considerable morbidity and mortality associated with LVAD infection as well as ongoing efforts to improve antimicrobial use, studies evaluating antimicrobial prophylaxis among LVAD recipients are lacking. Although this work is limited by the small sample size and the lack of site-specific infection rates from the participating centers, the results of this survey do demonstrate wide variability in SIP regimens across institutions underscoring the lack of consensus regarding best practices.

As a larger portion of LVAD use is shifted toward long-term or destination support, it is essential that the relative risks and benefits of different SIP approaches are critically evaluated. Although it is highly unlikely that a future randomized control trial will identify the optimal approach, individual centers can be better stewards of antimicrobials by closely monitoring their individual infection rates and the associated microbiology. Besides specific antimicrobial agents, efforts to ensure optimization of dosing strategies (e.g., administration within 1 hour before incision, redosing for prolonged operations, etc.) are a critical aspect of surgical site infection (SSI) prevention. In addition, active efforts to decrease the duration of prophylaxis should be promoted. Clinical teams providing postoperative care should carefully consider whether there is any real benefit to extending the duration of perioperative antimicrobials, particularly vancomycin. ${ }^{5}$ The use of automatic stop orders may also be helpful.

In addition to systemic antimicrobials, there may be a larger role for chlorhexidine to prevent device infection, particularly in terms of driveline infections. As noted above, 11 centers reported routine use of chlorhexidine. Recent studies demonstrate the benefits of chlorhexidine bathing among patients with central venous catheters, including those in the ICU setting as well as the long-term acute care hospital. ${ }^{7,8} \mathrm{~A}$ modified strategy may have efficacy in regards to SSI prevention among LVAD recipients, especially in regard to driveline infection. Future research should help determine which agents provide the best outcomes for patients while providing the fewest doses of broadspectrum therapy. Such studies should also consider potential decolonization strategies including those using chlorhexidine.

\section{REFERENCES}

1. Rose EA, Gelijns AC, Moskowitz AJ, et al: Randomized evaluation of mechanical assistance for the treatment of congestive heart failure (REMATCH) study group. Longterm mechanical left ventricular assistance for end-stage heart failure. N Engl J Med 2001;345:1435-1443.

2. Holman WL, Park SJ, Long JW, et al: Infection in permanent circulatory support: Experience from the REMATCH trial. J Heart Lung Transplant 2004;23:1359-1365.

3. Baddour LM, Bettmann MA, Bolger AF, et al: Nonvalvular cardiovascular device-related infections. Circulation 2003;108:2015-2031.

4. Edwards FH: The society of thoracic surgeons practice guideline series: Antibiotic prophylaxis in cardiac surgery, Part I: Duration. Ann Thorac Surg 2006;81:397404.

5. Harbarth S, Samore MH, Lichtenberg D, et al: Prolonged antibiotic prophylaxis after cardiovascular surgery and its effect on surgical site infections and antimicrobial resistance. Circulation 2000;101:2916-2921. 
6. Eyler RF, Butler SO, Walker PC, et al: Vancomycin use during left ventricular assist device support. Infect Control Hosp Epidemiol 2009;30:484-486.

7. Popovich KJ, Hota B, Hayes R, et al: Effectiveness of routine patient cleansing with chlorhexidine gluconate for infection prevention in the medical intensive care unit. Infect Control Hosp Epidemiol 2009;30:959963.

8. Munoz-Price LS, Hota B, Stemer A, et al: Prevention of bloodstream infection by use of daily chlorhexidine baths for patients at a long-term acute care hospital. Infect Control Hosp Epidemiol 2009;30:1031-1035. 